\title{
Polysaccharide-Based Hydrogels for Tissue Engineering
}

\author{
Jae-Won Lee, Hyo-Seok An, Kuen Yong Lee \\ Department of Bioengineering, Hanyang University \\ Seoul 133-791, Republic of Korea \\ jw136337@gmail.com; dksgytjr89@gmail.com; leeky@hanyang.ac.kr
}

\section{Extended Abstract}

Polysaccharide is one of widely utilized materials in biomedical areas due to its excellent biocompatibility. However, most polysaccharide-based hydrogels are typically prepared in the presence of excipient chemical reagents that may cause toxicity. In this presentation, we report how hyaluronate hydrogels can be prepared without toxic chemical cross-linking agents. Hyaluronate is composed of $\beta-1,4-\mathrm{D}$-glucuronic acid- $\beta-1,3-N$-acetyl-D-glucosamine residues, and is abundant in synovial fluid and extracellular matrix. We introduced alginate to the hyaluronate backbone, allowing gel formation in the presence of calcium ions [1]. The mechanical properties of hydrogels were varied depending on type and concentration of polysaccharide. Tissue regeneration using these hydrogels were carried out by subcutaneous injection of primary chondrocyte/hydrogel constructs into a mouse model.

In addition, a control of stem cell phenotype using hydrogels has been extensively investigated, and a use of cellinstructive polymers is critical to regulate the proliferation and differentiation of mesenchymal stem cells (MSCs) [2]. In this presentation, we report a biomimetic system to culture MSCs by providing stem cell niche using cell-instructive alginate microspheres. Chondrogenic differentiation of MSCs is typically influenced by aggregate formation [3] and cadherin is a key factor in mediating cell-cell interactions during stem cell condensation and chondrogenesis. Alginate microspheres, prepared by the water-in-oil emulsion method and solidified in the presence of calcium chloride [4], were thus modified with a peptide derived from E-cadherin receptor. Peptide-modified alginate microspheres formed an aggregate in the presence of MSCs, resulted in excellent cell viability, and promoted chondrogenesis in vitro. This approach may find useful applications in 3-D stem cell culture and tissue engineering.

\section{References}

[1] H. Park, E. Woo, and K. Y. Lee, "Ionically cross-linkable hyaluronate-based hydrogels for injectable cell delivery," J. Controlled Rel., vol. 196, pp. 146-153, 2014.

[2] M. B. Oliveira, W. Song, and J. F. Mano, "Development of an injectable system based on elastin-like recombinamer particles for tissue engineering applications," Soft Matter, vol. 7, pp. 6426-6434, 2011.

[3] S. Ravindran, A. McAlinden, and T. M. Hering, "Changes of chondrocyte expression profiles in human MSC aggregates in the presence of PEG microspheres and TGF-b3," Biomaterials, vol. 32, pp. 8436-8445, 2011.

[4] E. Woo, H. Park, and K. Y. Lee, "Shear reversible cell/microsphere aggregate as an injectable for tissue regeneration," Macromol. Biosci., vol. 14, pp. 740-748, 2014. 